\title{
Efecto crítico de la pandemia por covid-19 en el empleo de México
}

\author{
Fidel Olivera Lozano* \\ Guillermo Olivera Lozano**
}

Recepción: 27 de abril de 2021 / Aceptación: 9 de julio de 2021.

Resumen: Como efecto de la pandemia por covid-19, se analiza el ciclo del empleo formal durante 2020 en sus tres etapas: parálisis con destrucción, recuperación interrumpida y recaída. Asimismo, con datos del Instituto Mexicano del Seguro Social sobre el comportamiento mensual del número de trabajadores asegurados, se identifica el impacto de la gestión gubernamental y la evolución de los contagios en el empleo perdido o ganado en cada etapa. Se ilustra su comportamiento por sector de actividad, entidad federativa, tamaño de unidad económica, rango de salarios, sexo y grupo de edad. El mayor desempleo afectó los servicios turísticos y recreativos, las entidades más pobladas con áreas metropolitanas densas, las unidades económicas medianas y grandes; los trabajadores jóvenes, muy jóvenes y de más de 60 años, y los de peores salarios. La recuperación de los empleos preexistentes ocurrirá en 2022, pero su calidad tardará más tiempo en recuperarse. Es probable que el mercado laboral no vuelva a ser como antes.

PALABRAS CLAVE: covid-19, México, empleo formal, sector económico, estados.

\footnotetext{
* Profesor del Centro Regional de Investigaciones Multidisciplinarias de la U N A M, campus Morelos. Correo: olivera@crim.unam.mx O RCID : https://orcid.org/oooo-ooo2-4538-1682.

** Investigador en el Centro Regional de Investigaciones Multidisciplinarias de la U N A M, campus Morelos. Correo: gol@unam.mx o R C I D : http://orcid.org/oooo-ooo1-6978-9992.
}

doi: $10.32870 /$ cer.v0i128.7827 


\section{A Critical Effect of the Covid-19 Pandemic on Employment in Mexico}

Abstract The cycle of formal employment during 2020, following the Covid-19 pandemic, is analyzed in three stages: paralysis with destruction, interrupted recovery, and relapse. Likewise, using data from the Mexican Social Security Institute on monthly changes to the numbers of insured workers, it was possible to identify the impact of government management, and trace the history of infection provoked by the disease, in lost or gained employment at each stage. Changes to the numbers of those in employment are illustrated by the economic sector, state, size of economic unit, salary range, sex and age group of the workers. The highest rate of unemployment was in tourism and recreational services, the most populous states with dense metropolitan areas, medium and large economic units, among young workers, the very young and those over 60 years old, and those with the worst wages. It is estimated that it will take at least until 2022 for the quality of work to recover and the accumulated deficit to be restored. KEYWORDS: Covid-19, Mexico, formal employment, economic sector, states.

\section{Introducción}

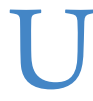

no de los principales efectos económicos de la pandemia por el virus S A R S - C O V-2 o covid-19, junto con el cierre de empresas y la caída del producto interno bruto (PIB ), fue la pérdida de empleos formales e informales a lo largo de 2020. El número de trabajadores registrados en el Instituto Mexicano del Seguro Social (I M s s) da cuenta de una disminución de 20482943 a 19773732 plazas laborales formales entre marzo y diciembre, lo que significó una pérdida neta de 709211 empleos. Si bien el saldo anual resultó por debajo de lo pronosticado por algunas fuentes, ${ }^{1}$ no deja de ser una debacle si se consideran los datos de subocupación y cambio en la participación laboral, que fueron especialmente elevados durante el primer confinamiento, ${ }^{2}$ o que hasta el primer semestre

${ }^{1}$ Como el Banco de México, que estimó una pérdida de hasta 1.4 millones de trabajos formales, o B BVA (2020), que la estimó en un millón y medio.

${ }^{2}$ Con base en datos de la Encuesta Telefónica de Ocupación y Empleo (Е т о в) del Instituto Nacional de Geografía y Estadística (INEG I), durante abril de 2020, el peor mes para el empleo, 2.1 millones de personas se convirtieron en desocupadas respecto a marzo del mismo año, tanto del sector formal como del informal. Este indicador se refiere a la población económicamente activa (P E A) que manifiesta no tener trabajo, pero que realiza acciones de búsqueda la semana previa a cuando se le consulta. 
de 2021 no se había recuperado el empleo perdido durante 2020. Más lejos aún han quedado los niveles de empleo de octubre de 2018, cuando se alcanzó un máximo de 20.7 millones de trabajos formales registrados.

Varias fuentes consideran que la recuperación económica y del mercado laboral en México se podrá lograr hasta 202302024 (Serrano, 2021), o incluso después, de acuerdo con el tipo de indicador que se utilice, por varias razones. La principal es que el país forma parte de América Latina, la región más afectada por la pandemia a escala internacional, según la Comisión Económica para América Latina y el Caribe y la Organización Internacional del Trabajo (Cepal y o Iт, 2020); aunado a que México venía de su primera caída del Р в в en diez años (-0.1\% en 2019), mientras que en Latinoamérica el quinquenio 2014-2019 fue una de las etapas de menor crecimiento en décadas (0.4\%). Otro argumento es que varios indicadores de México retrocedieron a la condición que tenían en 2018 o incluso antes, de manera que la «recuperación» esperada en 2021 no será suficiente para recobrar lo perdido. Respecto al crecimiento económico, por ejemplo, tras la caída de $-8.5 \%$ del P I в en 2020, se espera un rebote de $5.5 \%$ en 2021 ; pese a ello, sin embargo, el crecimiento genuino será de solo $1.8 \%$, por lo que los niveles previos a la pandemia se conseguirán hasta 2023. En el caso del p I в per cápita la recuperación se espera hasta 2026 (Morales, 2021).

A lo señalado se suman los efectos sociales, que también representan retrocesos sobresalientes. En primer término, se suman 9.8 millones de nuevos pobres (Coneval, comunicado $5,18 / 5 / 2021$ ), lo que sitúa el problema de la pobreza en las dimensiones existentes trece años atrás. La pobreza laboral, referida a la población con ingresos inferiores al valor de la canasta alimentaria, aumentó $3.8 \%$ entre el primer trimestre de 2020, cuando era de $35.6 \%$, y el primer trimestre de 2021 , que es del $39.4 \%$, situación que

Ahora que, si se considera la reducción en la participación laboral o caída de la PE A (personas ocupadas o desocupadas la semana previa a cuando se les preguntó si buscaron trabajo en caso de no tenerlo) en el mismo mes, esta se redujo en 12 millones, pues pasó de 57.7 millones de personas a 45.4 millones. La población subocupada, por su parte, aumentó en 5.9 millones (16.3\%). Debe tenerse en cuenta que la E T O E no es estrictamente comparable con la Encuesta Nacional de Ocupación y Empleo (E N OE), pero es una aproximación útil.

${ }^{3}$ De marzo de 2020 a junio de 2021 , aún faltaban por recuperarse 438156 empleos formales. Será necesario que transcurra todo el año para volver a la cifra de inicio de la pandemia, y parte de 2022 para regresar a los números de 2019. 
prevalecía en 2017, antes de los aumentos al salario mínimo. En materia de desigualdad, mientras tanto, con base en el índice de Gini, se ha retrocedido 15 años (Cepal y o I T, 2020; Morales, 2021). ${ }^{4}$

Adicionalmente, la capacidad de las unidades económicas para generar empleo se ha visto afectada. De acuerdo con el Estudio sobre la Demografía de los Negocios (U D N) del INEG I de 2020, por ejemplo, a diferencia del poco más de un millón de establecimientos que cerraron y tenían un promedio de tres personas ocupadas, los que se crearon en el mismo año lo hicieron con dos trabajadores en promedio.

En suma, el mercado laboral mexicano experimentó una crisis como nunca antes, que acentuó las condiciones de precariedad que ya lo caracterizaban. El reto que tiene por delante implica recuperar las plazas laborales perdidas y las condiciones relativas a la calidad del trabajo que también se vieron impactadas en mayor o menor medida, como los sueldos, los horarios y las prestaciones, que son las principales. A ello habrá que sumar el millón y medio de personas que desde 2020 han llegado a la edad de trabajar.

A raíz de lo descrito, este trabajo se propone analizar el comportamiento del empleo formal en 2020 durante el lapso transcurrido entre marzo, mes de inicio de la pandemia, y diciembre. El corte de tiempo es por razones prácticas, evidentemente, ya que el problema de desempleo seguirá manifestándose durante 2021 y parte de 2022 , vinculado a la evolución de la pandemia y a la conclusión con éxito del proceso de vacunación. La lenta recuperación del empleo en el primer semestre de 2021 así lo indica.

La fuente de información utilizada corresponde a los reportes mensuales del número de trabajadores registrados en el i M s s. Estos permiten no solo conocer la cantidad de empleos creados y perdidos cada mes, sino identificar también cómo se han comportado los trabajos perdidos o creados, según sea el caso, por sector económico, entidad federativa, tamaño de establecimiento, nivel de salario y sexo de los trabajadores implicados. Se dedica una sección a cada aspecto, antecedidos por una reseña de cómo distintos análisis consideran que la actual crisis ha trastocado el funcionamiento del mercado del empleo y de qué forma el gobierno federal podría reconducirlo a una situación deseable; y en segundo lugar, se desarrolla una caracterización de los tres periodos en que se divide el año 2020 en función del ciclo a que dio lugar la pandemia en la evolución del empleo formal. Estas dos secciones guían el resto del contenido de este artículo.

\footnotetext{
${ }^{4}$ Esto debido a un aumento del $5.6 \%$ del índice, lo que podría traducirse en una nueva década perdida, según la secretaria ejecutiva de la Cepal, Alicia Bárcena (Villanueva, 2021).
} 
Impacto inédito en la economía y el mercado laboral, y estrategias de superación que construir en un escenario aún incierto El consenso sobre la excepcionalidad de la crisis económica ocasionada por la pandemia por covid-19 es innegable, al igual que deben serlo las medidas para superarla en materia tanto de producción como de empleo, sin omitir, por supuesto, las medidas sanitarias ni la urgencia de completar la vacunación de todas las personas, para de esa manera controlar eventuales rebrotes. En los casos de México y de América Latina, es la peor crisis en 120 años, según la Cepal (2021).

En este tenor, es de esperar que la afectación de la crisis a la actividad económica y la calidad del empleo sean de mediana y larga duración, y que no se supere con la sola recuperación del P I B y los empleos perdidos, cuando eso pase. Es probable que el empleo formal retroceda temporalmente en favor de las ocupaciones informales, como ya ha sucedido en crisis o recesiones anteriores — entre ellas la de $2001-$, para recuperarse años después, si bien con una acentuada precarización porque ocurre en los estratos de menor ingreso $^{5}$ (Samaniego, 2018, 2019). Es factible, asimismo, que los salarios y el número de horas trabajadas se mantengan estancados temporalmente, de acuerdo con indicios que reporta la O I T (2020a, 2020b), tanto por la reapertura económica parcial como por la aceleración del proceso de robotización en ciertas actividades en el ámbito internacional. Este último aspecto, en específico, significará un proceso de transformación, destrucción y creación de empleos, con efectos a más largo plazo (Weller, 2020).

Las tendencias observables hasta el momento en México y América Latina son contrapuestas. Por un lado, efectivamente, se acentúan las características tradicionales de precariedad del empleo; por otro, se aceleran algunos procesos positivos que ya estaban en marcha, como el teletrabajo y el comercio en línea, que si bien no favorecen la contratación de trabajadores, impulsan una mayor productividad, elemento que a largo plazo se traduce en crecimiento, aumento de la renta per cápita y más alto nivel de vida (Jimeno, 2016).

En el caso de la precariedad y el desempleo, los trabajadores jóvenes son los más expuestos y tienen mayor riesgo de ver obstaculizado su ingreso al mercado laboral, lo que amenaza su futuro. La inserción laboral femenina está por debajo del promedio de los

${ }^{5}$ La precarización laboral va más allá de la informalidad — falta de prestaciones laborales — y los bajos salarios, e involucra contratos a tiempo parcial y de carácter transitorio o atípico, así como los esquemas de subcontratación, que tuvieron un auge en los últimos años (Samaniego, 2019). 
países más desarrollados, con lo que su aportación al crecimiento económico nacional se limita. Y la población adulta enfrenta rezagos de capacitación ante la demanda de mayor calificación en determinados sectores productivos. Mientras tanto, la mayor parte de los establecimientos económicos micro y pequeños, que representan de $90 \%$ a $95 \%$ de las unidades económicas, adolecen de acceso a financiamiento.

Dado lo anterior, ¿de qué estrategias y herramientas disponen los gobiernos para reconducir sus economías y recuperar el empleo, para de esa manera devolver los niveles de bienestar prepandémicos a su población? Las evidencias muestran capacidades divergentes con base en el nivel de desarrollo de los países; los mejor preparados son los desarrollados, seguidos por los emergentes y, por último, los países en vías de desarrollo. Esta divergencia aplica también para las empresas, los sectores económicos, las regiones y el tipo de trabajador, de acuerdo con su calificación. Algunos organismos internacionales que comparan datos entre países han propuesto estrategias de carácter general, aplicables a cualquier país.

En diversas publicaciones, la Cepal propone cuatro estrategias generales encaminadas a mantener o reanudar el trabajo, apoyar los hogares y las empresas, recuperar el crecimiento económico y producir localmente los insumos clave para la producción. Entre los contenidos específicos, propone mantener el trabajo a distancia y desarrollar nuevos modelos de negocio, reconvertir fábricas y generar nuevas capacidades laborales, así como recomponer las cadenas de suministro (Cepal y o I T, 2020; Cepal, 2020a, 202ob).

Dos de las propuestas que más hacen todo tipo de organismos internacionales y que aceptan muchos gobiernos han sido la transferencia directa de recursos económicos a personas u hogares y las medidas de alivio fiscal a las empresas. Estas acciones probaron ser exitosas en Europa occidental y Norteamérica, ya que los apoyos superaron con creces lo otorgado después de la gran crisis financiera internacional. En Latinoamérica, debido a lo limitado de sus ingresos públicos y al poco margen fiscal en general, los apoyos no han sido suficientes después de un año y han aumentado la deuda de varios países. México ha sido uno de los países que menos recursos ha destinado a ese fin en términos de proporción del P IB, lo que ha sido duramente criticado.

Por ello, para el subcontinente latinoamericano se ha planteado la necesidad de asegurar en forma emergente y temporal un ingreso mínimo equivalente a una línea de pobreza, fortalecer mecanismos de apoyo a los ingresos, empleos y micro, pequeñas y medianas empresas estratégicas, así como trabajar para que el acceso a un ingreso básico y un sistema de cuidados sea universal, como parte de un conjunto de derechos primordiales 
(Cepal y OIT, 2020; Cepal, 2020a, 2020b). En última instancia, sin embargo, se reconoce que la desigualdad, la pobreza, la poca inversión y la baja productividad económica son problemas estructurales que frenan su crecimiento (Cepal, 2021).

La Organización para la Cooperación y el Desarrollo Económicos (O E CD, 2019) advertía un año antes de la pandemia sobre algunas tendencias en el mundo del trabajo, que a raíz de esta adquieren mayor relevancia. Destaca al respecto su recomendación de llevar a cabo programas específicos de recalificación para adultos maduros, desempleados y trabajadores informales cuyas habilidades laborales ya no sean adecuadas; en segundo lugar, «mejorar el acceso a la enseñanza y la formación profesional». Se menciona también la conveniencia de facilitar la transición de trabajadores ocupados en industrias y regiones en declive a otras que aún tienen margen de competitividad.

Todo lo reseñado hasta aquí conlleva, en última instancia, el regreso del Estado como actor central del desarrollo económico y social a escala internacional pues, como ha quedado demostrado, su papel en la gestión de la pandemia ha sido fundamental para la paulatina superación de la misma, a pesar de los errores en que se incurrió en un principio. Este crédito ganado deberá servir para sacar adelante al mundo de la peor crisis económica y social contemporánea con el apoyo de organismos multilaterales.

A continuación se desglosan los efectos de la pandemia en el empleo formal del país.

Fases del ciclo del empleo en 2020:

parálisis y destrucción, recuperación interrumpida y recaída ${ }^{6}$

El saldo neto anual de la evolución del empleo formal en diciembre de 2020 fue de una pérdida de más de 700 ooo plazas laborales; su comportamiento, sin embargo, no fue lineal a lo largo del año. En lugar de ello se configuraron tres momentos, ligados a dos periodos de confinamiento forzoso y otro de confinamiento atenuado con reapertura productiva (véase gráfica 1 ).

El primer periodo de parálisis económica y destrucción manifiesta de empleos, que abarcó de abril a julio, ${ }^{7}$ fue resultado de un confinamiento social más o menos rígido, con

${ }^{6} \mathrm{El}$ análisis a lo largo del texto se hace en función de la relación entre periodos de confinamiento y comportamiento del empleo formal, vinculado a la evolución de la pandemia en el país.

${ }^{7}$ Las autoridades sanitarias de México declararon la Jornada Nacional de Sana Distancia el 23 de marzo de 2020, que tentativamente concluiría el 30 de abril, lo cual implicó una cuarentena de suspensión generalizada de actividades no esenciales. No obstante, debido a la evolución de la pandemia, la jornada 


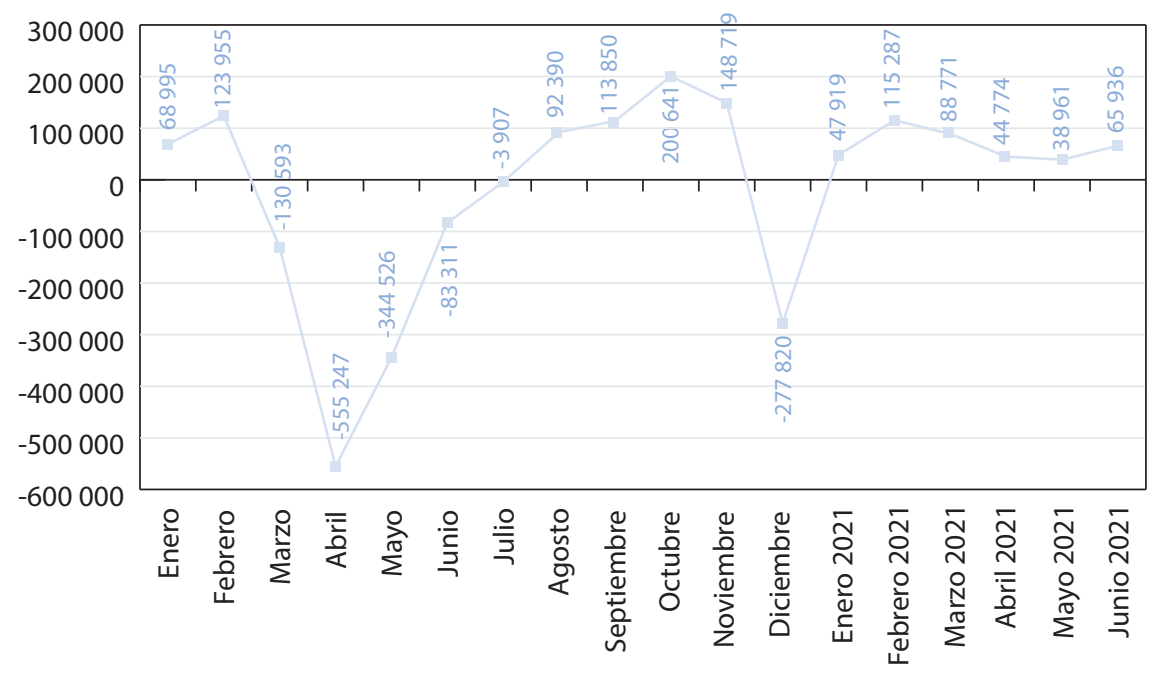

Fuente: Elaboración propia con datos del Instituto Mexicano del Seguro Social.

suspensión de actividades económicas no esenciales durante casi tres meses, pero cuyos efectos duraron cuatro meses, siendo abril y mayo los de peores resultados de todo el año, con el cierre de 899000 plazas. Se perdieron un total de 986991 empleos formales en el país durante este primer periodo.

El segundo periodo, de reactivación económica parcial y recuperación interrumpida de empleos, duró de agosto a noviembre e implicó el resarcimiento de 555600 empleos. ${ }^{8}$

se extendió hasta el 17 de mayo en municipios sin contagios registrados y hasta el 31 de mayo en todos los municipios donde existía registro de ellos; e incluso hasta el 15 de junio en la Ciudad de México. Así, a nivel nacional el 1 de junio inició la «nueva normalidad» mediante la instrumentación del semáforo epidemiológico, con la justificación de que el recurso del confinamiento estaba agotado y había tenido impacto negativo en la sociedad y la economía. El semáforo permitiría ser más selectivos en términos geográficos para no repetir un confinamiento de igual intensidad en todos los estados y municipios al mismo tiempo. La caída de empleos se atenuó en julio, pero continuó ya con muy baja intensidad.

${ }^{8}$ La instrumentación del semáforo epidemiológico permitió al gobierno federal hacer converger la gestión de la pandemia con la gestión económica. Tan pronto como se adoptó, el 1 de junio, reiniciaron 
La presión social y empresarial interna y externa en mayo y junio impulsaron la reanudación de actividades; inicialmente esto se reflejó en una disminución del número de empleos perdidos, y a partir de agosto, en los primeros saldos positivos del año. Coadyuvó a ello el control de la saturación de las unidades de cuidados intensivos de los hospitales. La reactivación no fue suficiente porque el aumento en la movilidad de la población en el último mes del año obligó a un nuevo confinamiento.

El tercer periodo, de recaída del empleo, correspondió al último mes del año. En diciembre se disparó la cantidad de contagios por covid-19 debido al incremento de la actividad comercial y de prestación de servicios en forma presencial. Así que, en previsión de las reuniones familiares esperadas por las celebraciones de fin de año, fue necesario establecer un segundo confinamiento generalizado, con suspensión nuevamente de actividades no esenciales en estados donde el semáforo epidemiológico cambió a rojo. En esta ocasión la reclusión fue tardía y se debió a que la conducta de la sociedad no se ajustó a lo requerido. Asimismo, la definición de «actividad no esencial» se fue haciendo más flexible ante la demanda de grupos empresariales específicos y la presión social. En este tercer período se perdieron 277830 empleos, lo que implicó el retroceso de la mitad de lo ganado entre agosto y noviembre. En total, de abril a diciembre de 2020 se perdieron en el país 709211 empleos formales.

Es importante mencionar que la pérdida de empleos de diciembre de 2020 resultó menor que la ocurrida en el mismo mes de 2019, cuando se perdieron 382210 puestos de trabajo formales. Incluso, es la menor cantidad de empleos perdidos en un mes de diciembre en los últimos cinco años. ${ }^{9}$

En síntesis, la distinción de estos tres periodos en relación con el comportamiento del empleo durante 2020 es relevante porque su impacto fue diferente en cada uno de ellos y configuran un claro momento de crisis del empleo y el mercado laboral en Méxi-

actividades las industrias automotriz, minera y de la construcción, así como la maquiladora. Fue el caso de la BMw en San Luis Potosí, Fiat-Chrysler en el Estado de México y Coahuila, Nissan en Aguascalientes, General Motors en Guanajuato y Coahuila, Ford en Sonora y Toyota en Baja California y Guanajuato. Esto sucedió cuando aún estaban en semáforo rojo varios estados, pues desde el 12 de mayo se les había considerado actividades esenciales. Solo en Puebla se mantuvieron cerradas estas actividades por decisión del gobernador. En el caso de la industria maquiladora, reanudó actividades el 1 de junio. ${ }^{9}$ La pérdida de puestos de trabajo de fin de año suele explicarse por las denominadas razones estacionales, pues en diciembre muchas empresas hacen ajustes a su plantilla laboral. 
co. Es de esperar que esta crisis, lejos de concluir con el fin de la pandemia, cuando ello ocurra, se prolongue por unos años más, pues va unida a un proceso de cierre definitivo de establecimientos económicos. Además, no solo será necesario recuperar el nivel de empleo del mes de inicio de la pandemia, sino el nivel de octubre de 2018, cuando empezó a registrarse una pérdida de dinamismo del mercado laboral; así como sumar la cantidad de trabajos que debieron crearse desde entonces hasta la fecha para incorporar a la nueva fuerza de trabajo resultante de la dinámica demográfica. Lo contrario implica el riesgo de que la destrucción del empleo se convierta en un problema estructural (Cervantes, 2020).

Colapso del sector turismo y declive de la construcción,

el comercio, los servicios sociales y la agricultura

Hay dos formas de expresar el comportamiento de la pérdida o generación de empleos en el corto periodo de tiempo que se analiza: una es a partir de datos absolutos y la proporción de cada sector productivo en relación con el total de la actividad económica, y otra con datos relativos en términos de crecimiento o decrecimiento porcentual de cada sector — respecto a sí mismo—, tomando como base 100 marzo, el mes de inicio de la pandemia en México. Así se procedió en el análisis que sigue.

Parálisis inicial generalizada de la economía y destrucción del empleo, abril-julio

En términos absolutos, durante el periodo abril-julio el sector que concentró la pérdida de empleos a nivel nacional fue el de servicios a empresas, personas y el hogar con -335000 ( $34 \%)$, que comprende las actividades relacionadas con el turismo principalmente (gráfica 2). En orden descendiente, le siguieron la industria de la transformación con-170 000 (17.3\%), agricultura-ganadería -130 000 (13.2\%), comercio-126 000 ( $12.8 \%$ ) y construcción - 103000 ( $10.5 \%)$. En conjunto, arrojaron la pérdida de 866000 trabajos (el $87.8 \%)$.

Tuvieron pérdidas menores, pero no por ello poco importantes, los servicios sociales y comunales con -71 00o (7.2\%), además de comunicaciones y transportes con -43000 (4.4\%); en la industria extractiva la disminución de trabajos fue de solo -6 753 . Por su parte, la industria eléctrica y de captación y suministro de agua potable, vinculada al aumento de consumo de los hogares en cuarentena, tuvo un pequeño aumento de 933 nuevos trabajadores.

En términos relativos, el decrecimiento que cada sector experimentó respecto a sí mismo, tomando como base su situación en marzo, indica que los servicios a las em- 


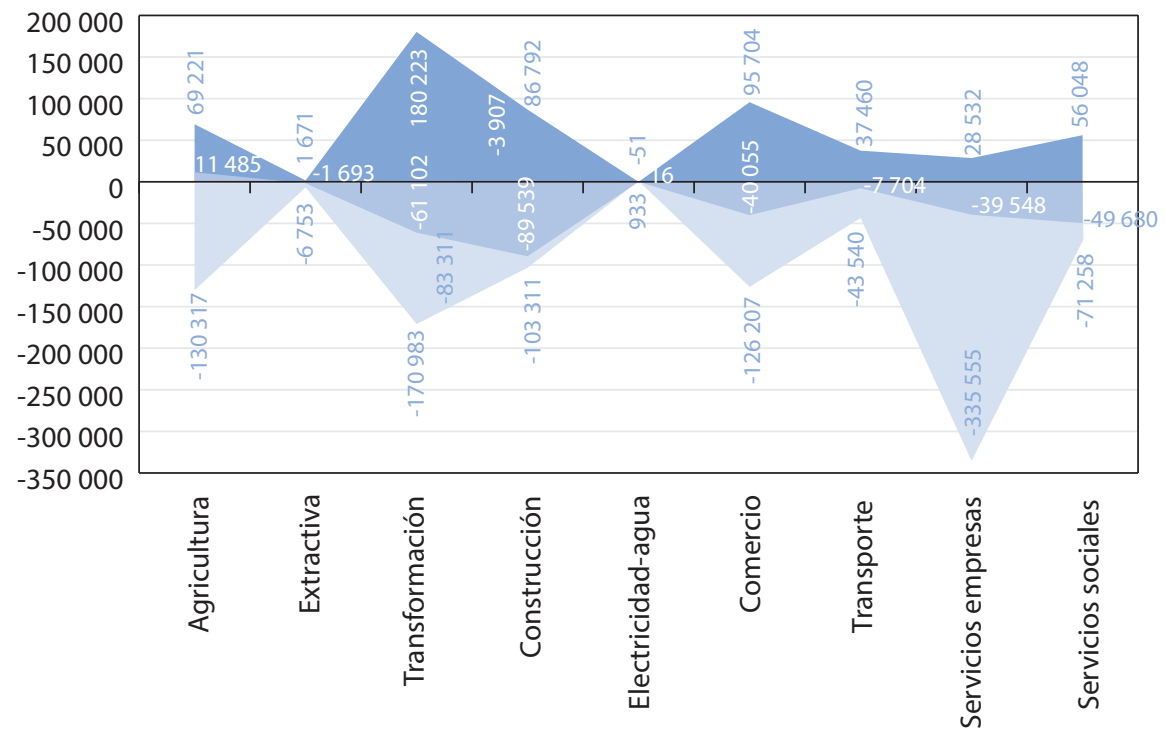

Abril-julio

Agosto-noviembre

Diciembre

Fuente: Elaboración propia con datos del Instituto Mexicano del Seguro Social.

presas, personas y el hogar no fue el primero sino el segundo con mayor cierre de plazas laborales (-7.0\%). En cambio, la agricultura-ganadería observó una reducción de -16.1 $\%$, y la industria de la construcción de $-6.5 \%$. La utilidad de estos datos es que ayudan a identificar los estados específicos donde se han presentado estos decrecimientos y, por lo tanto, tomar medidas de remediación más precisas.

Un ejemplo es la agricultura-ganadería, cuyo cierre de plazas laborales se concentró en Sinaloa ( -45000$)$ y Veracruz (-22 000), junto con Sonora y Jalisco (-1 7 ooo cada uno). $\mathrm{O}$ la industria extractiva, en la que el $54 \%$ del cierre de empleos ocurrió en únicamente tres estados: Zacatecas, Veracruz y Sonora.

\section{Reactivación interrumpida entre agosto y noviembre}

En el periodo agosto-noviembre hubo una notable generación de empleos (555 000) a escala nacional, aunque solo permitió recuperar $56 \%$ de los perdidos en los meses previos. Por sector, no obstante, la industria de la transformación recuperó los 170000 empleos perdidos y generó 10000 más. De los otros sectores, ninguno tuvo una recupera- 


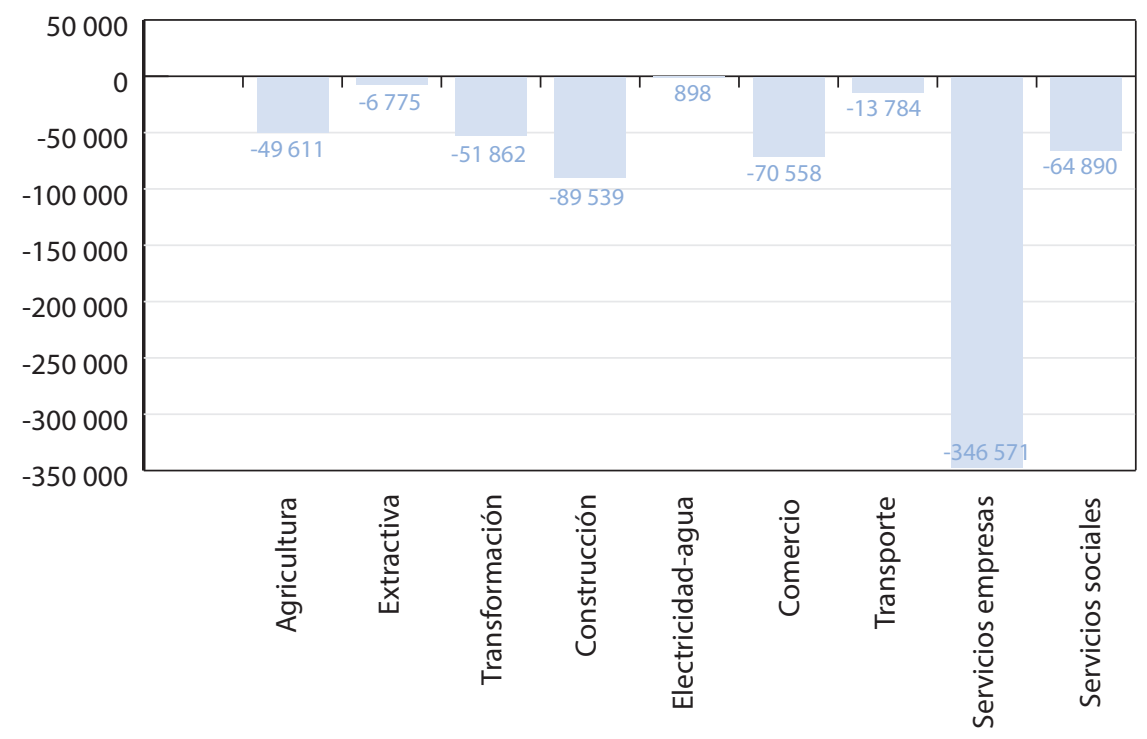

Fuente: Elaboración propia con datos del Instituto Mexicano del Seguro Social.

ción igual, pero el comercio (95 000), la construcción ( 86 ooo), los servicios sociales y comunales ( 56000 ) y los transportes y comunicaciones (34000) recuperaron entre $75 \%$ y $86 \%$ de lo perdido; la agricultura-ganadería recuperó $53 \%$ (69 000).

Que la industria manufacturera sea la que tuvo la mayor recuperación se debe a que está integrada a las cadenas productivas internacionales del sector; de manera destacada, la industria automotriz y de autopartes, al igual que la maquiladora. Destacaron, por lo tanto, los estados del centro occidente y el norte del país, además del Estado de México, en el proceso de reactivación económica.

Por otra parte, sin embargo, la recuperación temporal en dichos meses no fue generalizada en todas las entidades federativas. Al menos siete continuaron perdiendo empleos en agosto (Cervantes, 2020), como la Ciudad de México, Sonora, Nayarit y Chiapas, si bien en cantidades que promediaron los 2300.

\section{Recaída atada a la intensificación de la pandemia a fin de año}

En diciembre se registró un fuerte aumento en los contagios de covid-19, por lo que el semáforo epidemiológico en la mayoría de los estados donde se venía recuperando el $38 \mid$ 


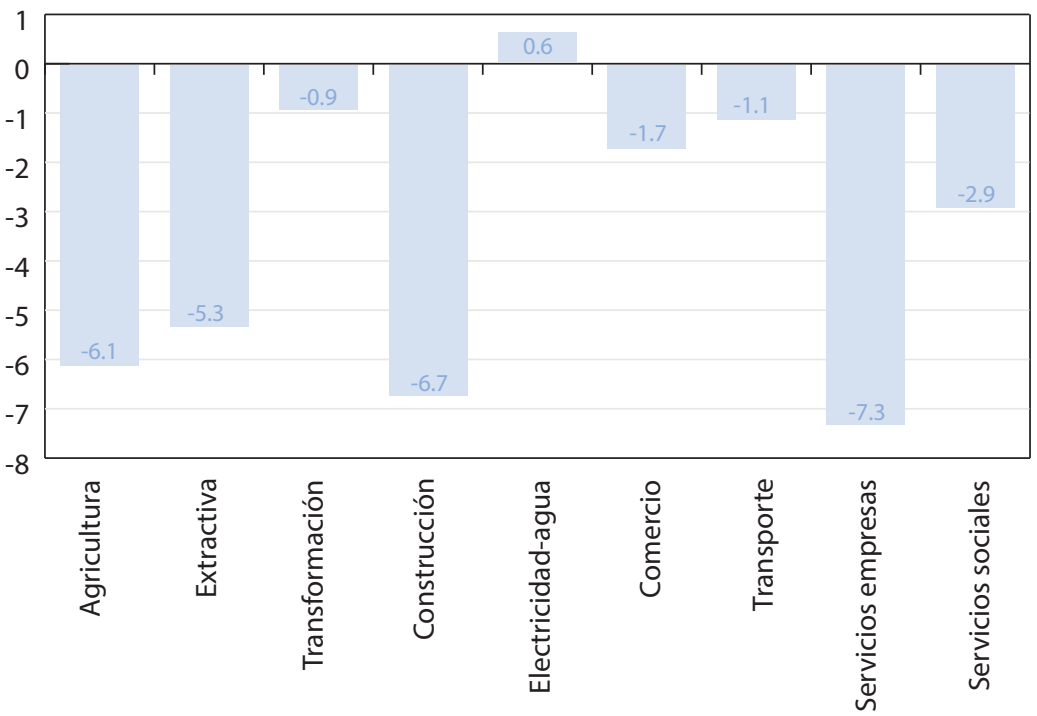

Fuente: Elaboración propia con datos del Instituto Mexicano del Seguro Social.

empleo cambió a rojo, lo cual implicó un nuevo confinamiento de la población y la suspensión de actividades no esenciales. Esto provocó que se perdiera la mitad del empleo creado entre agosto y noviembre. El mayor impacto absoluto lo tuvieron las industrias de la construcción (-89000) y de la transformación (-61 000), y el mayor impacto relativo correspondió a los servicios a las empresas, las personas y el hogar.

En el balance final de marzo a diciembre, la crisis del desempleo en México dio lugar a la debacle de los servicios a las empresas, las personas y el hogar — que incluye el turismo - por la desaparición de 346 ooo empleos ( $49 \%$ del total), así como al declive de la industria de la construcción, el comercio y los servicios sociales y comunales, con pérdidas de 106000,70000 y 64000 empleos, respectivamente (gráfica 3). En términos relativos, mientras tanto, el tercer sector que más decreció respecto a sí mismo, junto con los dos primeros mencionados, fue el de la agricultura, ganadería, silvicultura, caza y pesca $(-6.1 \%)$ (gráfica 4$)$.

Las ramas más afectadas, como parte de la actividad turística, fueron la preparación y el servicio de alimentos y bebidas, junto con los servicios de alojamiento temporal. En la industria de la construcción fueron las obras de infraestructura y la edificación de obra 
pública, así como las instalaciones sanitarias, eléctricas, de gas y aire acondicionado. En cuanto al comercio, fueron la compra venta de máquinas, muebles, aparatos e instrumentos para el hogar, sus refacciones y accesorios; la venta al menudeo de prendas y accesorios de vestir y artículos para su confección; la compra venta de alimentos, bebidas y productos de tabaco; la compra venta de papelería, útiles escolares y de oficina; y libros, periódicos y revistas.

En sentido contrario al de su marcado declive inicial, la industria de la transformación mostró resiliencia al final, por lo que es de esperar que a lo largo de 2021 mejore su desempeño general, impulsado por su carácter transnacional y apoyado en parte por reglas gubernamentales más flexibles. Finalmente, solo hubo un sector con saldo positivo — aunque ínfimo - : el de electricidad, gas y agua, por el aumento de su demanda a causa del confinamiento de la población.

Impacto territorial en entidades de alta densidad urbana, asentamientos metropolitanos y economía diversificada o altamente especializada En términos territoriales, la crisis del desempleo formal a lo largo del año tuvo como protagonistas de primer orden las cuatro entidades con mayor aportación al P I B total nacional (Ciudad de México, Jalisco, Nuevo León y Estado de México), ${ }^{10}$ donde se encuentran las zonas metropolitanas más importantes del país. ${ }^{11} \mathrm{Su}$ estructura económica está diversificada y su densidad urbana es elevada.

En un segundo orden, sobresalieron entidades con una fuerte base industrial localizadas en el centro (Guanajuato, Querétaro y Puebla) ${ }^{12}$ y el norte del país (Baja California, Sonora, Chihuahua y Coahuila), ${ }^{13}$ con presencia ya sea de industria automotriz o maquiladora de exportación.

${ }^{10}$ Estas cuatro entidades generaban el $41 \%$ del PIB nacional en 2019.

${ }^{11}$ Zonas metropolitanas de la Ciudad de México, 20.8 millones de habitantes; de Guadalajara, 4.9 millones, y de Monterrey, 4.7 millones. Además de las zonas metropolitanas de Toluca y de Tianguistenco, en el Estado de México, y las de Ocotlán y Puerto Vallarta en Jalisco. Esto de acuerdo con la delimitación de zonas metropolitanas de 2015 (Segob, Sedatu, Conapo e IN E G I, 2018).

${ }^{12}$ Puebla contiene la cuarta zona metropolitana más grande del país, con 2.9 millones de habitantes en 2015. En Guanajuato sobresalen las zonas metropolitanas de Celaya, Guanajuato, León y MoroleónUriangato; en Querétaro está la zona metropolitana de Querétaro.

${ }^{13}$ En estos estados están las zonas metropolitanas de Mexicali, Tijuana, La Laguna, Monclova-Fronte$40 \mid$ 
En una tercera condición, cabe destacar el estado de Quintana Roo como caso representativo de la afectación al sector turístico, Sinaloa por el impacto negativo inicial en su sector agropecuario, y Veracruz, que es la tercera entidad más poblada del país y la quinta en aportación al Р г в. Los dos primeros estados se caracterizan por una alta especialización económica en los sectores en que más se vieron afectados.

\section{Fase destructiva sumamente concentrada}

Durante el periodo inicial de destrucción del empleo, seis estados concentraron 53.9 $\%$ de los casos: los cuatro que generan más riqueza y cuyas economías están más diversificadas en general, junto con el estado turístico por excelencia, que es Quintana Roo; el sexto es Sinaloa, debido a su especialización económica en actividades agropecuarias. En los casos de Ciudad de México (-178 ooo empleos), Jalisco (-89 000), Nuevo León (-76 000) y Estado de México (-59 000), su participación porcentual en el empleo total que se perdió es, en términos generales, equivalente al peso de cada una de estas entidades en el P I в nacional, aunque el empleo perdido superó ligeramente el peso económico de cada entidad; es decir, tuvieron un leve excedente de pérdida de empleos. Se puede atribuir tal situación a la diversificación de sus economías, que tienen presencia importante de los cuatro sectores más castigados: servicios a las empresas, las personas y el hogar; servicios sociales y comunales; e industrias de la transformación y de la construcción, además del comercio.

Como ejemplo, la Ciudad de México y los estados de México, Jalisco, Nuevo León y Guanajuato sufrieron la pérdida de $59.7 \%$ de los empleos dados de baja en la industria de la transformación; otro tercio desapareció en Coahuila, Baja California, Puebla y Querétaro. En el sector comercio, los cinco primeros estados mencionados experimentaron, asimismo, la pérdida de $66.2 \%$ de las plazas laborales, y junto con Quintana Roo, $51.8 \%$ en la construcción. Por último, los cuatro primeros más Quintana Roo y Baja California Sur perdieron $63.4 \%$ de los empleos en servicios a las empresas, las personas y el hogar; el séptimo lugar fue para Veracruz.

En suma, la pérdida de empleos durante la primera fase de la pandemia se concentró en seis y diez estados, dependiendo del sector de actividad.

ra, Piedras Negras, Saltillo, Chihuahua, Delicias, Hidalgo del Parral, Juárez, Guaymas, Hermosillo y Nogales. 


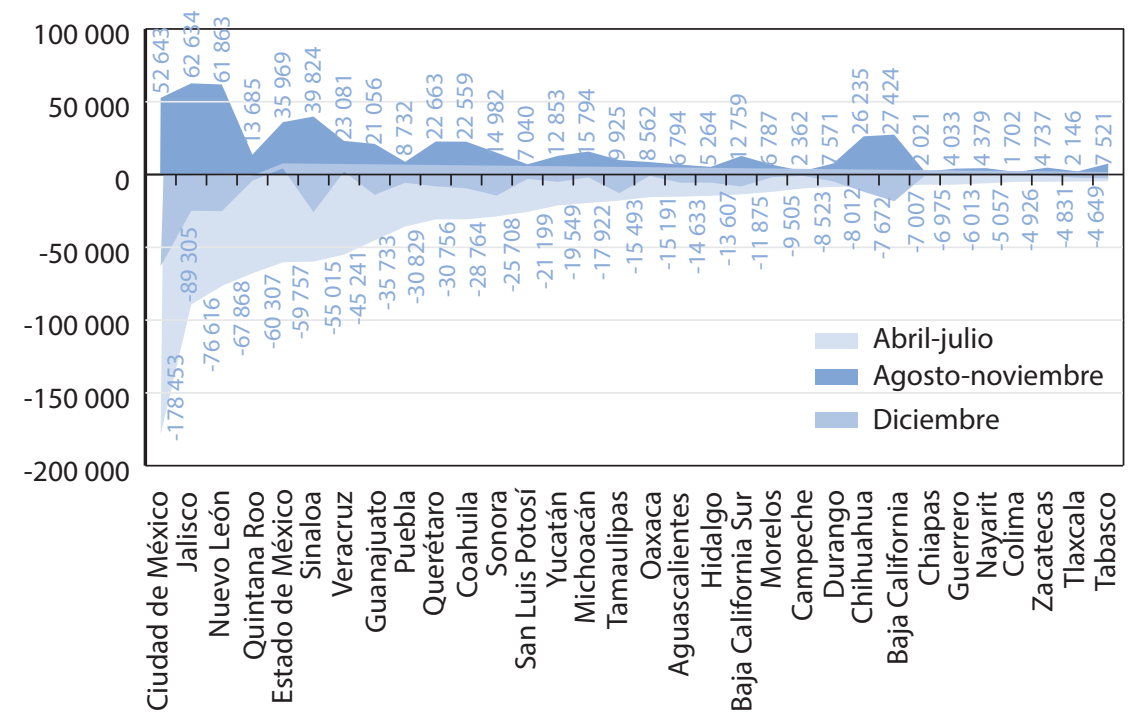

Fuente: Elaboración propia con datos del Instituto Mexicano del Seguro Social.

Recuperación interrumpida, impulsada por entidades más pobladas o industrializadas

De agosto a noviembre se recuperaron $64 \%$ (555 000) de los empleos perdidos en la fase previa. Siete estados encabezaron este proceso con $55 \%$ del total; los cuatro con mayor aportación al P I B, además de Sinaloa, Baja California y Chihuahua. En un siguiente grupo de seis estados, aunque con valores inferiores, sobresalieron Coahuila y Sonora, en el norte del país, y Guanajuato y Querétaro, en el centro-norte, además de Veracruz y Michoacán (gráfica 5).

En las entidades norteñas, la industria de la transformación fue la principal dinamizadora de la recuperación del trabajo, encabezadas por Nuevo León, en sintonía con lo ocurrido a escala nacional. En el Estado de México la reactivación de la industria se combinó con la del comercio; y algo parecido ocurrió en Jalisco, donde a la industria se le sumaron la agricultura, el comercio y actividades relacionadas con el turismo. En Sinaloa la agricultura y la ganadería recuperaron 25000 de los 45000 empleos cerrados previamente.

$\mathrm{Al}$ no reactivarse el sector turismo, no se recuperó el empleo en Quintana Roo, lo que se repitió en Nayarit, Baja California Sur y Guerrero. Jalisco fue el mejor posicionado en ese caso, pero aun así solo recuperó 10 ooo empleos de los 23 ooo perdidos en los meses anteriores en el sector de servicios a las empresas, las personas y el hogar. 
Como anotación final, todos los estados, sin excepción, recuperaron parte del empleo perdido en el periodo previo, pero todos lo hicieron en una cantidad insuficiente.

\section{Recaída de fin de año y saldo anual}

Además de los cuatro principales, el resto de los estados de la frontera norte experimentaron en diciembre el mayor impacto de la recaída en el cierre de plazas laborales, al igual que Guanajuato ( $79 \%$ de la pérdida total en diez estados). Salvo Coahuila, su participación en la pérdida del trabajo fue mayor que la de su participación en el p I в nacional, por lo que de nuevo son casos de exceso de pérdida. La Ciudad de México fue un caso extremo, lo que se explica porque estuvo un mayor número de semanas en confinamiento.

Por último, y como saldo anual, cabe destacar que tres entidades resultaron con saldo positivo (gráfica 6). En efecto, Chihuahua, Baja California y Tabasco terminaron el periodo de análisis con 6 334, 1350 y 263 empleos creados, respectivamente. Entre las entidades perdedoras, mientras tanto, la Ciudad de México, con 188 ooo trabajos menos, concentró $26 \%$ de la pérdida total del país. Le siguieron Quintana Roo (8.2\%), Jalisco ( $7.3 \%)$, Estado de México (6.5 \%) y Nuevo León ( $5.6 \%$ ), que juntos sumaron $54.2 \%$ del

GRÁFICA 6. México: pérdida neta de empleos por estado, marzo-diciembre de 2020

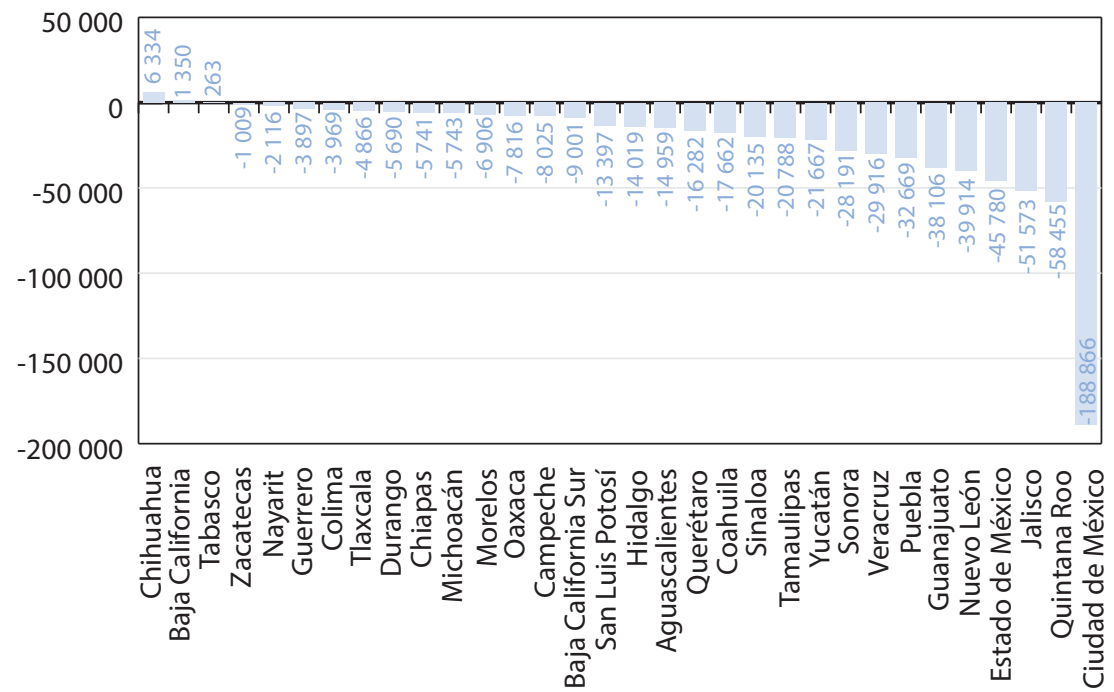

Fuente: Elaboración propia con datos del Instituto Mexicano del Seguro Social. 


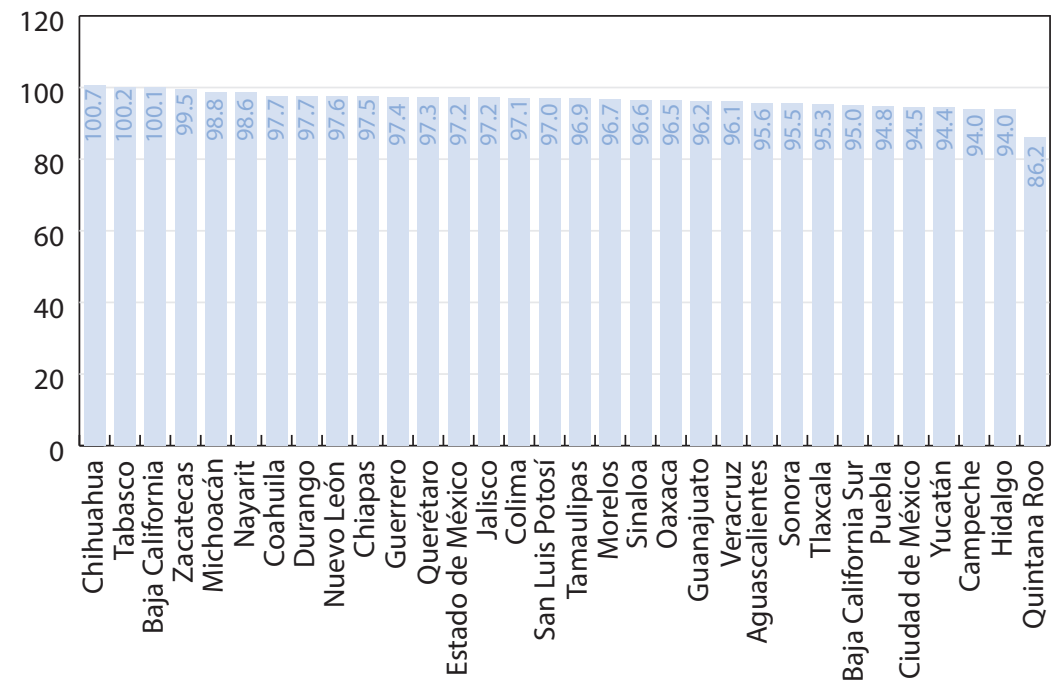

Fuente: Elaboración propia con datos del Instituto Mexicano del Seguro Social.

total de plazas laborales perdidas. Como ya se indicó, son las entidades más densamente pobladas por la presencia de áreas metropolitanas, así como diversificadas o altamente especializadas en términos económicos.

Las ramas de actividad más castigadas en la Ciudad de México fueron los servicios profesionales y técnicos; preparación y servicio de alimentos; servicios de alojamiento temporal; compra venta de equipo de cómputo o de procesamiento electrónico de datos y sus periféricos, con servicios de instalación, y compra venta de prendas y accesorios de vestir y artículos para su confección, entre los principales. En el rubro de manufactura sobresalieron la confección de prendas de vestir; las industrias editoriales, de impresión, encuadernación y conexas, y la fabricación de productos de hule.

Finalmente, el saldo del (des)empleo en cada estado en diciembre, respecto a su situación en marzo, que indica el decrecimiento ocurrido en ellos, coloca a Quintana Roo como la entidad más perjudicada por la pandemia, ya que perdió casi $14 \%$ de su planta laboral. Lejos de tal condición, le siguieron Hidalgo, Campeche, Yucatán, Ciudad de México, Puebla y Baja California Sur, con un decrecimiento del $6 \%$ al 5 \% (gráfica 7 ). 
Riesgo laboral incrementado para los trabajadores

de ingresos muy bajos y bajos, estabilidad para los de mayores salarios

Precariedad y segmentación como rasgo principal

Otro aspecto notable de la pandemia es el relativo a su impacto sobre el desempleo según rango salarial de los trabajadores. En este rubro en particular, el comportamiento del desempleo fue claramente dicotómico de abril a diciembre, ya que solo dos de los cinco rangos de ingresos en que se organizó la información resultaron fuertemente perjudicados: el de los trabajadores con ingresos mayores a un salario mínimo y hasta dos (rango 2$)^{14} \mathrm{y}$ el de los de ingresos mayores de dos y hasta cinco salarios mínimos (rango 3 ).

En estos dos rangos ocurrió $93 \%$ de la pérdida de trabajos en el periodo abril-julio (-926 000) y $96 \%$ de los perdidos en diciembre (-266 00o); en el lapso de recuperación agosto-noviembre se recobraron $86 \%$ (479 0oo) de los existentes en marzo (gráfica 8 ). En sentido opuesto, los empleos en un rango de ingreso de más de cinco y hasta diez salarios mínimos (rango 4) y el de más de diez salarios mínimos (rango 5 ) solo participaron con $3.5 \%$ de las pérdidas entre abril y julio $(-35000)$ y $2.3 \%$ en diciembre $(-6495)$. Entre

GRÁFICA 8. México: evolución del empleo según remuneraciones, abril-diciembre de 2020

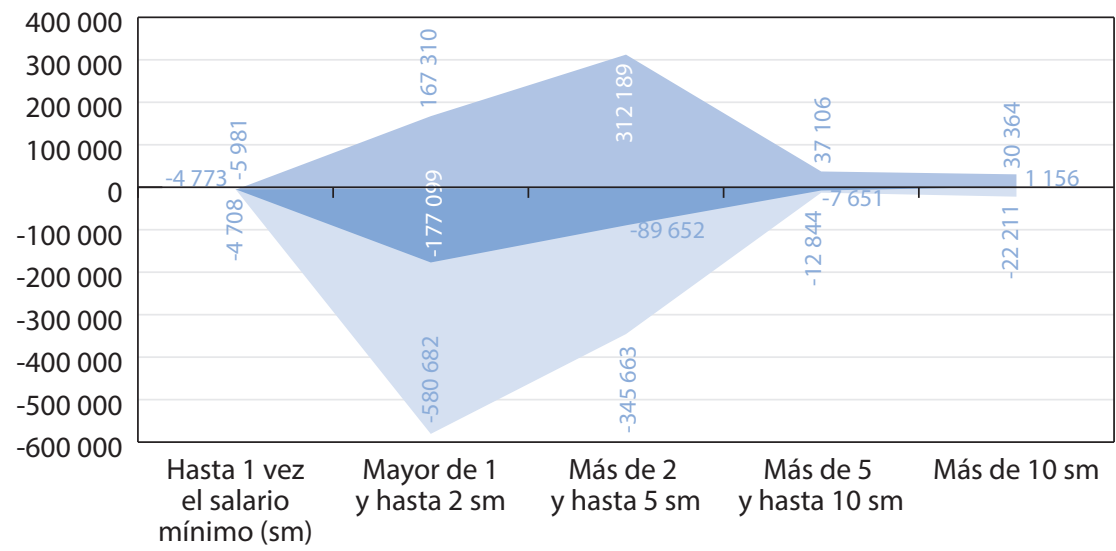

Abril-julio

Agosto-septiembre

Diciembre

Fuente: Elaboración propia con datos del Instituto Mexicano del Seguro Social.

${ }^{14}$ El rango 1 corresponde a los trabajadores que perciben hasta un salario mínimo. 


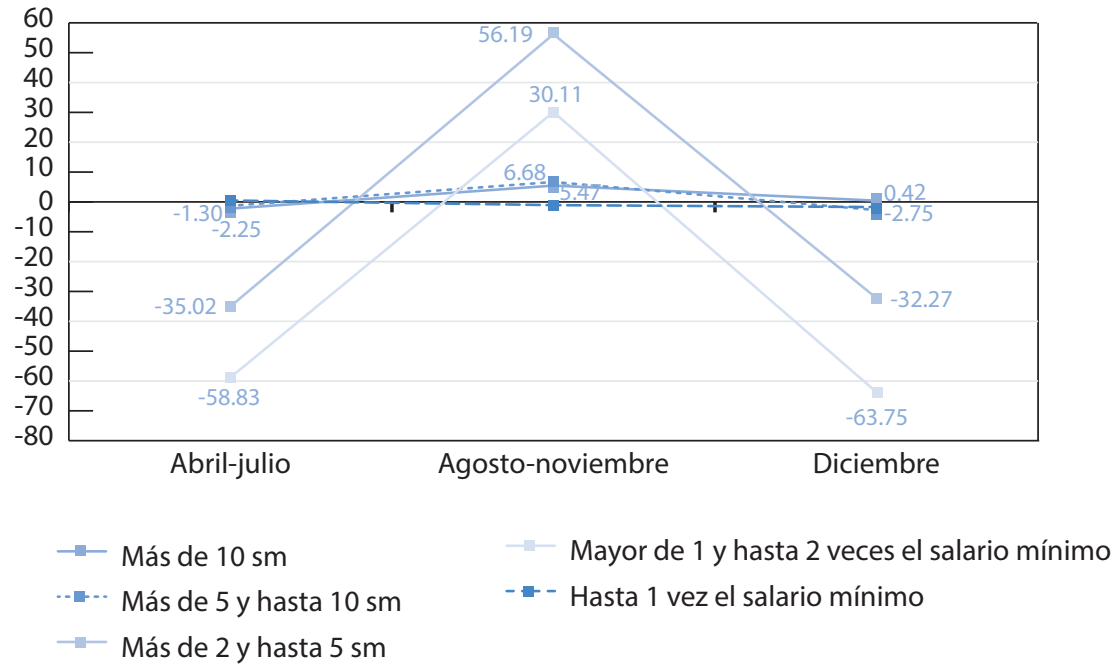

Fuente: Elaboración propia con datos del Instituto Mexicano del Seguro Social.

agosto y noviembre lograron duplicar lo perdido previamente con la creación de 67000 puestos de trabajo, equivalentes al $12 \%$ del total nacional. Un dato relevante adicional es que en diciembre los empleos en el rango de más de diez salarios mínimos incluso tuvieron un incremento de 7600 plazas. Esta estabilidad también la tuvieron los empleos con hasta un salario mínimo.

Como saldo final, de marzo a diciembre los rangos 2 y 3 perdieron 713000 plazas laborales, y los rangos 4 y 5 tuvieron un saldo positivo de casi 26000 empleos. Dicho en todas sus palabras, la crisis laboral producida por la pandemia se convirtió en una catástrofe para los trabajadores del sector formal con ingresos muy bajos y bajos (excepto para aquellos con ingresos de hasta un salario mínimo). Los trabajadores de ingresos medios y altos experimentaron la crisis, en todo caso, como una recesión. Adicionalmente, los rangos 2 y 3 han experimentado una marcada inestabilidad, en tanto que para los rangos 4 y 5 la estabilidad en el contexto de crisis generalizada es evidente (gráfica 9).

\section{Segmentación laboral según sector económico}

Los datos disponibles permiten también distinguir que los sectores económicos más afectados por el desempleo (véase el segundo apartado de este artículo) fueron, eviden$46 \mid$ 
temente, aquellos en los que se resintió más el impacto en los trabajadores con ingresos bajos y muy bajos; no obstante, hay algunas particularidades relevantes (gráfica 10). La primera es que en las industrias de la construcción y de la transformación los empleos con salarios de rango 2 (muy bajos) fueron los únicos castigados en el saldo anual; y la segunda, que todos los empleos de los demás rangos de salario tuvieron un saldo anual positivo. Un segundo aspecto es que en comercio y en servicios a las empresas, las personas y el hogar todos los rangos de salarios, sin excepción, cerraron empleos; pero hay que hacer notar que en comercio no hay prácticamente registro de empleos con salarios en el rango 5 (altos). En tercer término, el de agricultura y ganadería es un sector que principalmente paga salarios muy bajos, y bajos, como la construcción; por consiguiente, tuvo un fuerte impacto negativo durante el año con el cierre de plazas laborales en esta categoría de sueldos. Por último, en servicios sociales y comunales, los empleos en los

GRÁFICA 10. México: evolución del empleo formal según rango salarial y sector de actividad, marzo-diciembre de 2020

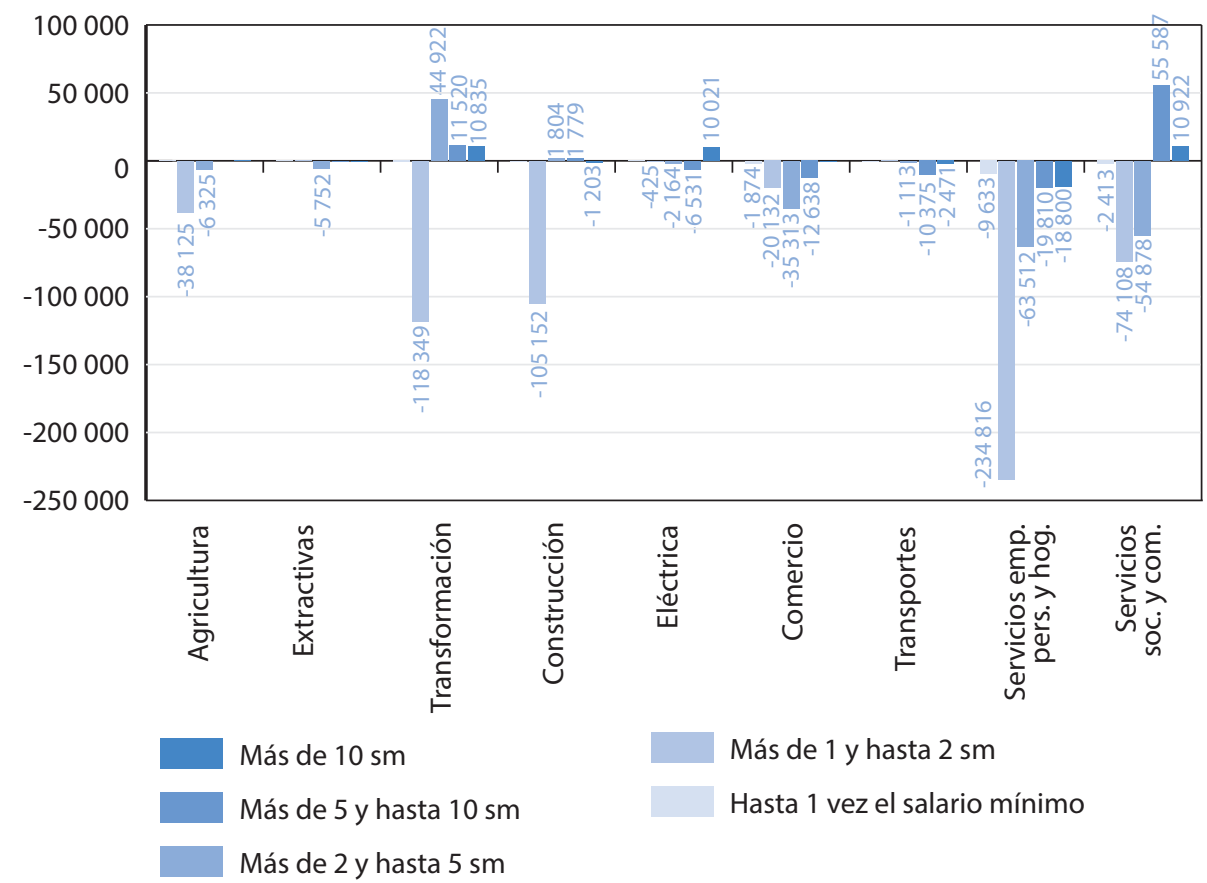

Fuente: Elaboración propia con datos del Instituto Mexicano del Seguro Social. 


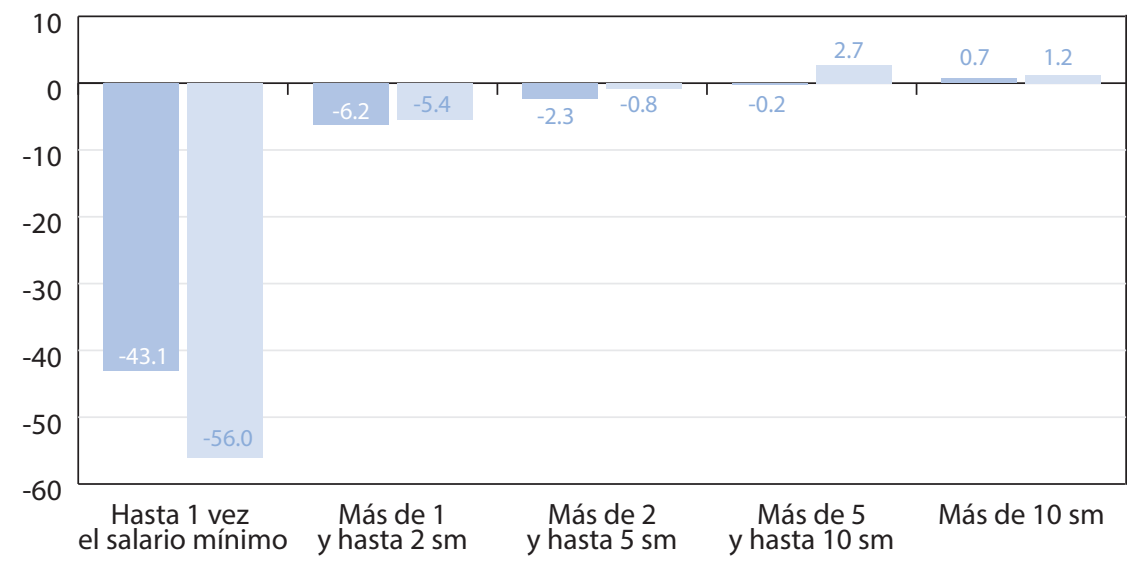

Mujeres

Hombres

Fuente: Elaboración propia con datos del Instituto Mexicano del Seguro Social.

rangos de salarios 4 y 5 fueron los únicos con saldo anual positivo notable; los rangos 2 y 3 aglutinaron las pérdidas del año.

\section{El desempleo femenino por rango salarial}

En cuanto a la distinción de trabajadores por sexo, teniendo en cuenta el inicio de la pandemia en marzo y que $61.7 \%$ de los trabajadores registrados en el IMss eran hombres y $38.3 \%$ mujeres, estas últimas se vieron perjudicadas con el desempleo en el rango 1 de salarios (hasta un salario mínimo) en $13 \%$ más que sus contrapartes masculinos. ${ }^{15}$ Salvo este caso, en los demás rangos de salarios su afectación fue menor. En los rangos superiores (4 y 5 ) su saldo anual incluso fue positivo y claramente mejor que el de los hombres (gráfica 1 1). Se debe aclarar que los datos solo expresan el crecimiento o decrecimiento del empleo según sexo y rango de salario respecto a su situación en marzo, ${ }^{16}$ por lo que

\footnotetext{
${ }^{15}$ Se debe tener presente que los empleos en este rango de salarios solo representan alrededor de $1 \%$ del total, por lo que su dimensión es poco significativa, excepto por el hecho de la distinción por sexo del personal y que no es favorable para las mujeres.

${ }^{16}$ En trabajos donde se contabiliza el desempleo incluyendo el sector informal sí se reporta mayor pér$48 \mid$
} 
no tienen que ver con la inequidad salarial existente y que registran otras fuentes de estadísticas.

Los servicios y el comercio, sectores feminizados

$\mathrm{Al}$ cierre de 2020, el $67.7 \%$ de los empleos perdidos correspondieron al sexo masculino y $32.2 \%$ al femenino, lo cual indica que los primeros tuvieron una sobrerrepresentación de $6 \%$ en el desempleo nacional. Por gran división económica, por otra parte, las actividades primarias (agricultura-ganadería) y las secundarias (extracción, transformación, construcción, y electricidad y agua $)^{17}$ están dominadas por trabajadores masculinos, con

GRÁFICA 12. México: pérdida de empleo por sector de actividad y sexo, marzo-diciembre de 2020

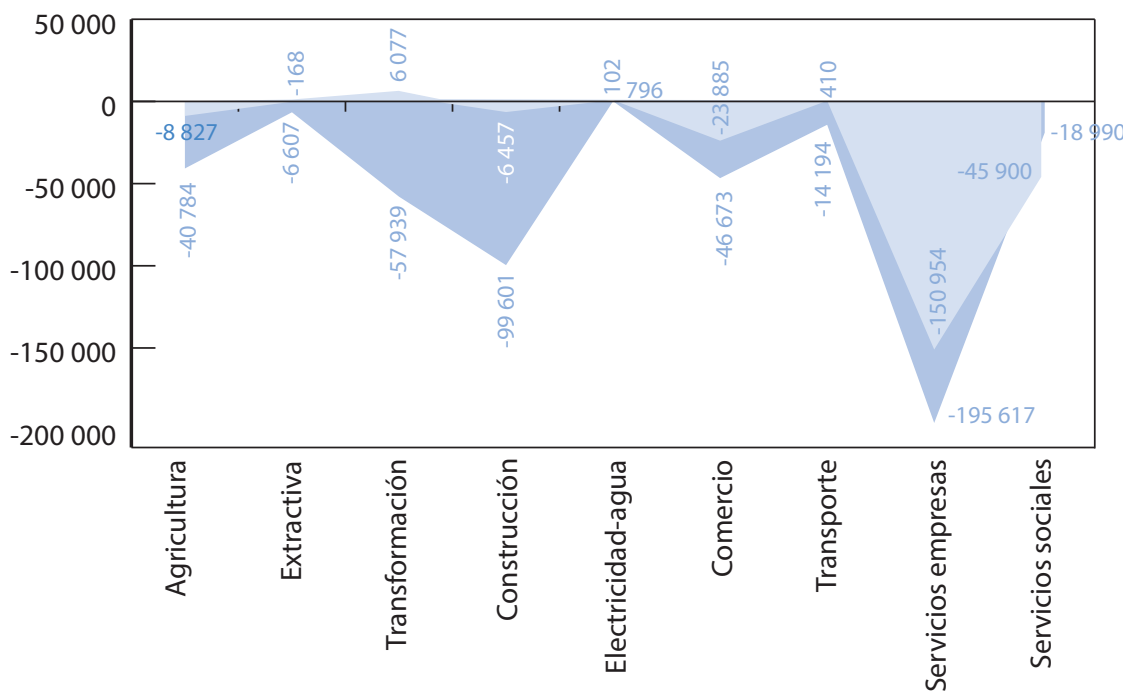

Hombre

Mujer

Fuente: Elaboración propia con datos del Instituto Mexicano del Seguro Social.

dida de empleo entre mujeres que entre hombres ( $3 \%$ superior), asociada a su concentración en ramas de actividad «feminizadas» como el servicio doméstico, restaurantes y hoteles, y comercio, o por su mayor implicación en el cuidado de familiares (Cepal y OIT, 2020).

${ }^{17}$ La participación de los hombres en el empleo formal por sector de actividad, en los sectores en que es dominante (por arriba del promedio nacional de $61.7 \%$ ), de mayor a menor, era de la siguiente ma- 
la consecuente «masculinización» del desempleo en ellas. Las divisiones de comercio y servicios, por el contrario, tienen una distribución más equilibrada, incluso con predominio femenino en los servicios sociales y comunales ( $57.6 \%)$; en comercio los hombres constituían $56.5 \%$ de la fuerza laboral, y en servicios a las empresas, las personas y el hogar $61.7 \%$, en los dos casos por debajo del promedio nacional.

Es por ello que, como se muestra en la gráfica 12, el desempleo femenino se aglomeró en el comercio, los servicios a las empresas, las personas y el hogar, y en los servicios sociales y comunales. En estos últimos, $70.7 \%$ de las plazas perdidas (45 900) estaban asignadas a mujeres, lo cual indica que fueron muy castigadas en este sector.

Destrucción laboral en la pequeña, mediana y gran empresa, y sobrevivencia de la unidad familiar

Los establecimientos económicos o empresas también resintieron la crisis de manera diferenciada a lo largo del año, según su número de trabajadores, elemento que define su tamaño. ${ }^{18}$ Los contrastes respecto a su participación en el desempleo producido son muy marcados. Por ejemplo, los de un solo trabajador, «que no pueden parar», no solo no cerraron sus operaciones, sino que generaron casi 7 ooo trabajos nuevos en el mismo número de establecimientos como saldo neto (gráfica 13).

El segmento de los microestablecimientos (de dos a cinco trabajadores), que suelen ser negocios familiares, aunque perdió 10709 empleos, puede considerarse que se mantuvo a flote pues solo aportó $1.5 \%$ de las plazas laborales cerradas. El volumen mayoritario de empleos perdidos (-305 000) se produjo en los establecimientos medianos (de 51 a 250 trabajadores), con poco más de dos quintas partes, y en los grandes ${ }^{19}$ (251 y más

nera al inicio de la pandemia: industria extractiva (90\%), construcción ( $84.4 \%)$, transporte y comunicaciones $(78.6 \%)$, electricidad y agua $(77.8 \%)$, agricultura-ganadería $(73.9 \%)$ e industria de la transformación $(63.1 \%)$.

${ }^{18}$ El I N E G I (2014) clasifica las empresas en cuatro tamaños (micro, pequeña, mediana y grande) con base en su número de trabajadores y el monto de sus ventas anuales. Los rangos, sin embargo, varían entre los tres únicos sectores que considera: industria, comercio y servicios. Estos criterios, por lo tanto, no pueden aplicarse en este trabajo porque abarca nueve divisiones económicas y la fuente de los datos es el I m s s. De ahí que se utilizó una agrupación ad hoc de las unidades económicas para distinguirlas por su tamaño en función solo de su número de trabajadores.

${ }^{19}$ Del 1 de abril al 31 de diciembre de 2020, 52 de 91 grandes empresas que cotizan en la Bolsa Mexi$50 \mid$ 


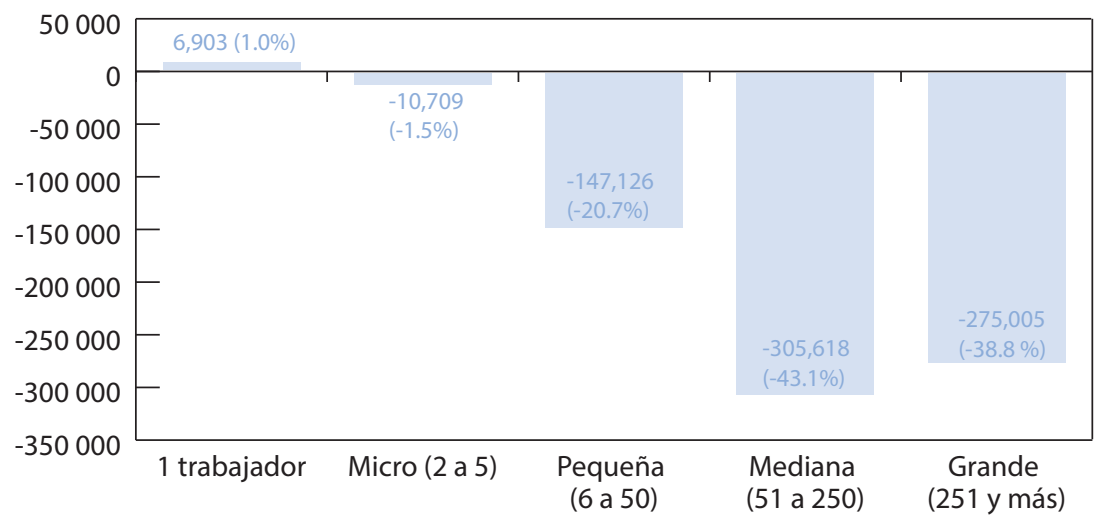

Fuente: Elaboración propia con datos del Instituto Mexicano del Seguro Social.

trabajadores) (-275 000), con poco menos de dos quintas partes. Las unidades económicas pequeñas (de seis a 50 trabajadores) participaron con $20 \%$ de los despidos, lo que también es una cantidad apreciable (-147000).

Un dato adicional es el que relaciona el tamaño de la unidad económica con cada uno de los sectores de actividad. Al respecto, los registros del I M s s indican que en la agricultura la desocupación a causa de la pandemia se produjo casi en su totalidad en empresas grandes, sobre todo de 1000 trabajadores y más (-67\%), y fue de $-32 \%$ en medianas; los demás tamaños de empresas tuvieron números positivos. Un comportamiento similar fue el de la industria extractiva, donde la gran empresa contribuyó con prácticamente todos los empleos perdidos y la pequeña tuvo una aportación mínima. Fue en los servicios a las empresas, las personas y el hogar, sin embargo, donde la gran empresa acumuló la mayor parte de la pérdida de empleos, y lo mismo sucedió con las pequeñas y medianas.

cana de Valores despidieron a 87995 trabajadores, equivalentes al $3.5 \%$ de su planta laboral. El $77 \%$ de este recorte se realizó en solamente diez firmas: Operadora de Restaurantes Alsea (-16 501$)$, Elektra (-14 740), Walmart (-10 366), Soriana (-6 938), Gigante (-5 525), Grupo Posadas (-4 121), Aeroméxico (-2 639), Grupo México (-2 608), América Móvil (-2 497) y Conglomerado Alfa (-2 026). Por su porcentaje de ajuste, C I E, Sports World, Proteak Uno, Grupo Hotelero Santa Fe y C M R recortaron entre $25 \%$ y $39.2 \%$ su base laboral (elfinanciero.com.mx). 
GRAFICA 14. México: pérdida de empleo por tamaño de unidad económica y sector de actividad, marzo-diciembre de 2020

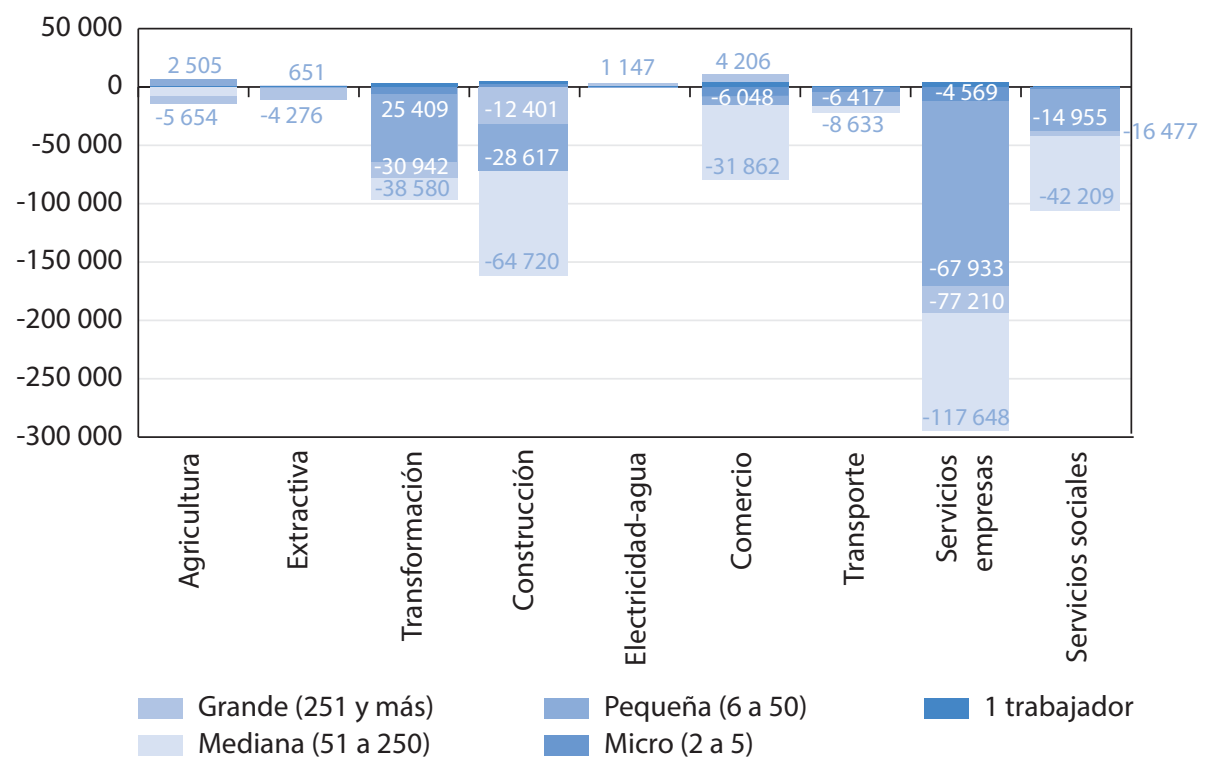

Fuente: Elaboración propia con datos del Instituto Mexicano del Seguro Social.

GRÁFICA 15. México: decrecimiento del empleo por tamaño de establecimiento, marzo-diciembre de 2020 (\%)

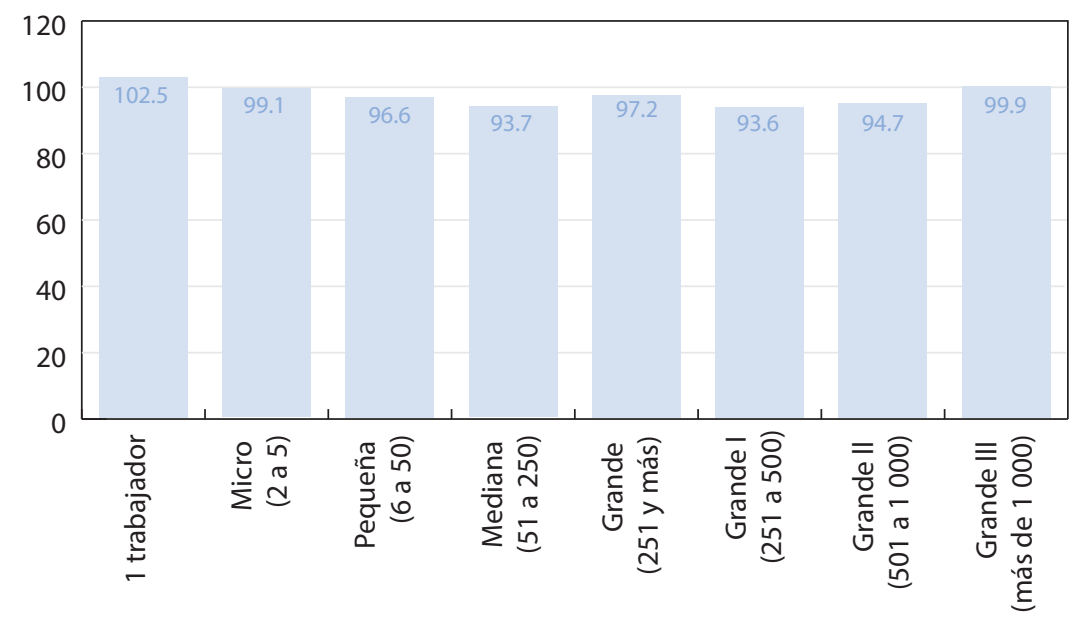

Fuente: Elaboración propia con datos del Instituto Mexicano del Seguro Social. 
En montos menores, pero en proporciones parecidas a lo anterior, se replicó en las industrias de la construcción y de la transformación, así como en los servicios sociales y comunales (gráfica 14).

Un hecho relevante adicional es que el desempleo en comercio se dio principalmente en empresas medianas. Finalmente, las unidades económicas de un trabajador tuvieron saldo positivo en todos los sectores económicos.

Ahora bien, a pesar de lo hasta aquí descrito, hubo sectores en los que la gran empresa tuvo saldo positivo, pues generó empleos nuevos en el saldo final del año. Fueron los casos del comercio (4 206) y la electricidad y agua ( 1147 ).

Para concluir, en la gráfica 15 se muestra el decrecimiento porcentual de empleos por tamaño de los establecimientos a lo largo del año. Los descensos más visibles ocurrieron en la mediana y gran empresa; de esta última, en los establecimientos de 251 a 500 trabajadores; en tanto que el segmento de 1000 y más trabajadores se mantuvo muy estable. Las unidades económicas de un solo trabajador incluso crearon $2.5 \%$ de los empleos nuevos.

Impacto superior del desempleo en los trabajadores más jóvenes

En números absolutos, el desempleo afectó superlativamente al grupo de edad de 20 a 29 años, puesto que de representar $28.9 \%$ del total de empleos existentes en marzo, aportaron $35 \%$ de la pérdida en el saldo neto final del año (-250 00o) (gráfica 16). Destaca,

GRÁFICA 16. México: pérdida de empleo formal por rango de edad del trabajador, marzo-diciembre de 2020

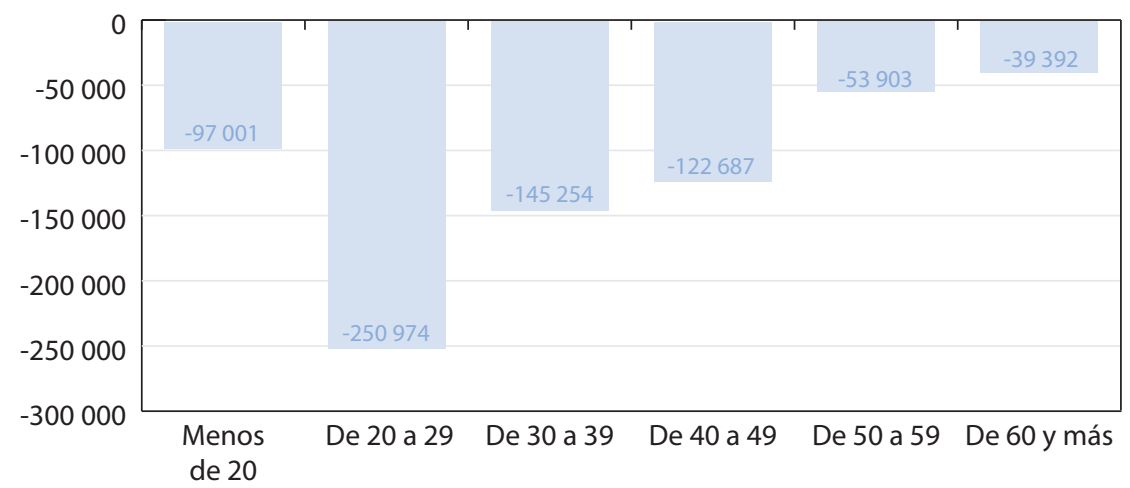

Fuente: Elaboración propia con datos del Instituto Mexicano del Seguro Social. 
GRÁFICA 17. México: decrecimiento del empleo formal por rango de edad del trabajador, marzo-diciembre de 2020 (\%)

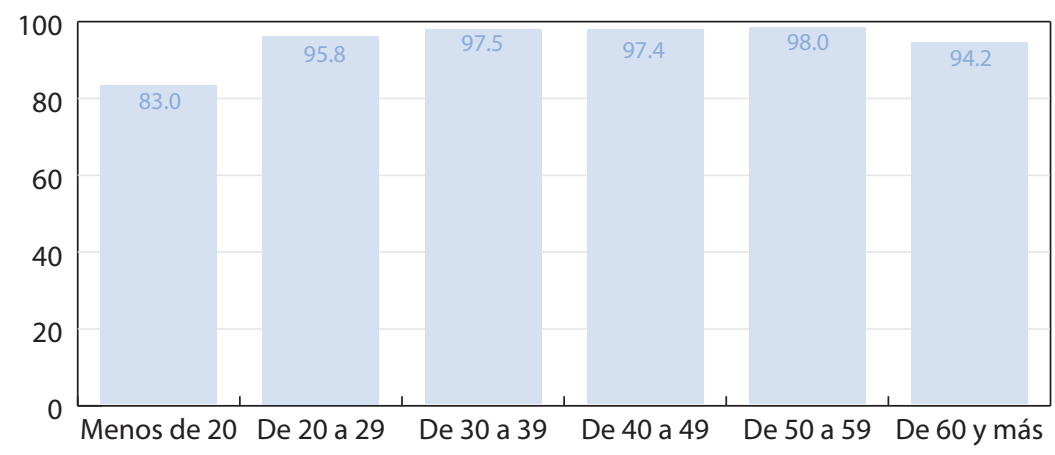

Fuente: Elaboración propia con datos del Instituto Mexicano del Seguro Social.

con este mismo criterio, el grupo de menos de 20 años, que participó con $13.7 \%$ de los empleos desaparecidos (-97 00o), siendo que solo representaba $2.8 \%$ de los empleos existentes en marzo. Por lo que, en términos relativos, los jóvenes de menos de 20 años fueron los que más resintieron el desempleo a nivel nacional (gráfica 17).

Los otros dos rangos de edad con fuertes pérdidas de trabajos, en términos absolutos, fueron los de 30 a 39 y los de 40 a 49 años de edad, con -145000 y - 122 0oo, respectivamente; aunque en una proporción cercana a la que tenían originalmente, de manera que su decrecimiento porcentual no fue significativo.

En otras fuentes que aportan información complementaria a la aquí incluida se menciona que el grado de escolaridad de los trabajadores está relacionado con la propensión al desempleo durante la pandemia. De manera que entre aquellos con educación básica se redujo hasta $15.1 \%$ su participación en el empleo, mientras que entre los que tienen estudios universitarios su contracción solo fue de $0.3 \%$ entre el primer y tercer trimestre de 2020. Para el cuarto trimestre incluso ya había nuevos trabajos para las personas con estudios superiores (Arceo y Guzmán, 2021). Estas últimas se ocupan en la administración pública, educación, salud, ciencia, seguros, finanzas y otras actividades profesionalizadas, lo que facilitó en muchos casos el teletrabajo y evitó así su exposición a la enfermedad por covid-19 (Cepal y o I T, 2020). Muchos de los jóvenes menores de 20 años se encuentran en la situación opuesta, de insuficiente formación académica, con el efecto de tener plazas laborales no consolidadas. 


\section{Consideraciones finales}

La pandemia por covid-19 ha tenido un impacto sin precedentes en el mercado laboral mexicano y, en general, en la actividad económica y el desarrollo social, de lo cual destacan varios aspectos que pueden reseñarse de la siguiente manera. Primero, el evento sorprendió al país en una fase de bajo crecimiento económico, baja inversión productiva y estancamiento en la creación de empleos formales. De ahí que, con un predominio del trabajo informal, sin prestaciones ni acceso a servicios adecuados de salud, y mucho menos a seguro de desempleo, las personas enfrentaron un cierre casi total de la economía en el segundo trimestre de 2020 que las dejó en condiciones de total vulnerabilidad. Este grupo de población experimentó las peores consecuencias en su economía y se expuso al peligro de contagio por covid-19.

Los trabajadores formales, por su parte, mostraron una clara segmentación del mercado de trabajo en función del grupo de edad afectado, el monto de sus salarios, su grado de escolaridad e incluso el carácter temporal o permanente del empleo. La mayoría de los trabajadores que perdieron su empleo fueron los jóvenes y muy jóvenes (seguidos por los de más de 60 años), los de salarios bajos y muy bajos y aquellos con baja escolaridad. Por el contrario, los trabajadores de 30 a 59 años, con ingresos medios y altos y de mayor escolaridad experimentaron mayor estabilidad; parte de ellos pudieron realizar con éxito teletrabajo, de acuerdo con su sector de ocupación.

Lo anterior no quiere decir que los trabajadores formales que conservaron su trabajo hayan estado exentos de afectaciones notables; entre ellos hubo quienes vieron reducidas sus jornadas laborales, y con ello sus ingresos. Peor, desde luego, fue la situación de los desempleados, que debieron recurrir al uso de sus afores como seguro de desempleo y que de abril de 2020 a abril de 2021 sumaron 23000 millones de pesos, de acuerdo con la Comisión Nacional del Sistema de Ahorro para el Retiro (Consar). Como es conocido, estos retiros reducen los ahorros y las semanas de cotización de los trabajadores con vistas a su jubilación.

El segundo aspecto es que las unidades económicas enfrentaron la crisis con diferente estrategia, obligadas por la debacle económica. Las pequeñas, medianas y grandes ajustaron el tamaño de su planta productiva, sus horarios y las remuneraciones para mantenerse a flote. Los microestablecimientos solo aportaron $1.5 \%$ del desempleo total, en tanto que los de un solo trabajador crearon casi 7 ooo nuevos trabajos; son negocios para los que no hubo otra opción que resistir. En ambos casos, no obstante, quedan efectos por resolver, como las deudas contraídas, falta de liquidez y la recuperación del mercado. 
México se caracterizó, a nivel América Latina, por el bajo nivel de apoyos fiscales otorgados a la planta productiva. Así que aun cuando eventualmente se recuperen en 2021 los empleos perdidos en 2020, quedará pendiente resolver los aspectos señalados, y también recuperar el nivel de empleo de 2018.

Cabe mencionar que un efecto inesperado, sobre todo para las medianas y grandes empresas, es que se obtuvieron avances en el desarrollo de nuevos canales de comercialización, y se demostró que en algunas ramas de actividad económica el trabajo es viable, por lo que es de esperarse que persistan dichas modalidades en el futuro.

En tercer término, se ilustró a lo largo del texto que sectores económicos con mayor interacción personal, como el comercio y los servicios, fueron de los más afectados, junto con otros que durante el primer confinamiento fueron considerados actividades no esenciales en varias de sus ramas de actividad, como en la industria de la transformación, o en todas, lo que ocurrió en la industria de la construcción. En el cuanto al comportamiento territorial de la pérdida de empleos, se mostró que hubo concentración en no más de diez estados del país, encabezados por los de mayor participación en el P I в y más población, circunstancias expresadas en una alta densidad urbana, asentamientos metropolitanos y economía diversificada en unos casos, como la Ciudad de México, el Estado de México, Jalisco y Nuevo León, y en otros casos muy especializada, como Quintana Roo en turismo. Misma situación que enfrentaron estados con industria maquiladora en el norte o industria automotriz en el centro-norte del país.

Finalmente, aunque se espera que pasen dos o tres años más para que la crisis en México sea superada, el país tiene como ventaja principal, respecto al resto de América Latina, por ejemplo, la vinculación tan fuerte de su sector externo con Estados Unidos. El programa de inversión del gobierno de ese país, los estímulos económicos a la planta productiva y la población, junto con el avance de la vacunación, se han traducido ya en la reactivación de la industria manufacturera. Esto se manifestará en una recuperación desigual en el país: avanzarán más rápido los estados donde se realice la vacunación más eficientemente y el semáforo epidemiológico avance hacia el color verde, y que al mismo tiempo tengan un sector exportador de mayor peso, sobre todo a Estados Unidos. Como las actividades económicas de mayor interacción personal tienen que ver con turismo, recreación y cultura, los estados y ciudades con alta dependencia de ellas podrían tardar más en recuperarse, a menos que se vacune a la población lo más pronto posible. 


\section{Referencias}

Arceo, E. y Guzmán, K. (2021). Mercado laboral en México: el saldo del primer año de la pandemia por covid-19. México: Observatorio Económico México Cómo Vamos, A.C.

Cervantes, D. (2020). Empleo formal se derrumba en abril, se pierden dos años de creación de empleos. B B VA Research, 13 de mayo. [bbvaresearch.com].

Cervantes, D. (2020). Claroscuros en la creación de empleo en agosto. B B VA Research, 30 de septiembre [bbvaresearch.com].

Comisión Económica para América Latina y el Caribe (Cepal) (2020a). Informe especial covid-19 $N^{\circ}$. El desafío social en tiempos del covid-19. Santiago de Chile: Cepal. [repositorio.cepal.org]. Comisión Económica para América Latina y el Caribe (Cepal) (202ob). Informe sobre impacto económico de América Latina y el Caribe de la enfermedad por coronavirus (covid-19). Santiago de Chile: Cepal [cepal.org].

Comisión Económica para América Latina y el Caribe (Cepal) (2021). La paradoja de la recuperación en América Latina y el Caribe. Crecimiento con persistentes problemas estructurales: desigualdad, pobreza, poca inversión y baja productividad. Santiago de Chile: Cepal [repositorio. cepal.org].

Comisión Económica para América Latina y el Caribe (Cepal) y Organización Internacional del Trabajo (о I T) (2020). Coyuntura laboral en América Latina y el Caribe. La dinámica laboral en una crisis de características inéditas: desafíos de política. Santiago de Chile: Cepal, o i T. [ilo. org].

Gobierno de México (2018). Delimitación de las zonas metropolitanas de México 2015. México: Secretaría de Gobernación, Secretaría de Desarrollo Agrario, Territorial y Urbano, Consejo Nacional de Población, Instituto Nacional de Estadística y Geografía [gob.mx].

Instituto Nacional de Estadística y Geografía (I N E G I) (2014). Censos Económicos 2014. Micro, pequeña y mediana empresa. Estratificación de los establecimientos. Aguascalientes: I N E G I . Jimeno, J. (2016). Crecimiento y empleo. Una relación turbulenta e incomprendida. España: R B A Libros.

Morales, Y. (202 1). Economía mexicana tardará un lustro en regresar al P I B per cápita precrisis: F M I. El Economista, 8 de enero [eleconomista.com.mx].

Organisation for Economic Co-operation and Development (O E CD) (2019). Employment outlook 2019. The future of work. Paris: O E C D.

Organización Internacional del Trabajo (о I T) (2020a). América Latina y el Caribe: tasas de participación, ocupación y desocupación nacional por año, según país y tramo de edad, 2010-2020. Sistema de Información y Análisis Laboral de América Latina y el Caribe (s I A L C ). Lima: o I T. 
Organización Internacional del Trabajo (O I T) (2020b). Formación profesional en la respuesta a la crisis y en las estrategias de recuperación y transformación productiva post covid-19. Nota técnica regional. Panorama laboral en tiempos de la covid-19 [oitcinterfor.org].

Samaniego, N. (2018). El desafío del empleo y los salarios. México: Consejo Nacional de Ciencia y Tecnología, El Colegio de México, Centro Tepoztlán, Foro Consultivo Científico y Tecnológico, A.C.

Samaniego, N. (2019). Los retos del empleo en una estrategia de desarrollo incluyente. Economía UNAM, 16(46), $217-227$.

Serrano, C. (2021). La demanda interna no reacciona: permanece débil. B BVA Research, 8 de abril [bbvaresearch.com].

Villanueva, D. (2021). Insuficiente, el rebote de 2021 para compensar la caída en A L . La Jornada, 17 de marzo [jornada.com.mx].

Weller, J. (2020). La pandemia del covid-19 y su efecto en las tendencias de los mercados laborales. Santiago de Chile: Cepal. 